\title{
Fighting fire with fire
}

\section{A co-infection model of two pathogens in a nematode yields insights into the complex inter- action between the microbes.}

The immune system of a healthy person will swiftly and effectively deal with opportunistic pathogens. Not so for those with a compromised immune system, for example, after severe trauma or invasive surgery. In these patients opportunistic infections pose a severe health risk and can be fatal.

Two pathogens causing such opportunistic infections, the gram-negative bacterium Acinetobacter baumannii and the yeast Candida albicans, have gained notoriety for causing increased outbreaks in intensive care units of hospitals. Both can survive the harsh environment of an intensive care unit, both thrive on plastic tubing used for catheters, and both colonize the respiratory tract of their victims.

Anton Peleg from the Massachusetts General Hospital, and with a history in Acinetobacter sp. research, was looking for an animal model to investigate the pathogenicity of bacteria when he met Eleftherios Mylonakis, an expert in fungal biology who had set up a model in Caenorhabditis elegans to study yeast infection in the worm.

The two scientists collaborated to replicate what is often seen in a hospital setting, the simultaneous exposure to both pathogens. They co-infected worms with $A$. baumannii and C. albicans, and made the unexpected discovery that the pathogens were antagonists.

In a monomicrobial infection model, the yeast transforms into a filamentous form after entering the worm and kills it by destroying its organs. In the co-infection model Peleg and Mylonakis observed prolonged survival of the worms; the bacteria were keeping the yeast in check, partly by killing it outright and partly by preventing its transformation into the filamentous form.

How exactly $A$. baumannii interferes with the yeast is not known, but a screen of about 600 A. baumannii transposon mutants yielded an exciting hit, a gene that is an important virulence regulator in other gram-negative bacteria and is involved in secretion. Peleg and Mylonakis plan to look in more detail at the molecular mechanism behind the bacteria's successful attack on the yeast.

C. albicans in turn is not a helpless victim of the bacteria: given an opportunity to develop into the filamentous form, it retaliates. Peleg explains: "Candida in a biofilm environment produces more of a quorumsensing molecule called farnesol that inhibits the bacteria."

With this co-infection in worm the researchers modeled the beginning of pathogen interactions in a host. By shedding light on the pathways that C. albicans uses to protect itself against A. baumannii, and vice versa, therapeutic targets may come to light.

The worms live longer as a result of the pathogens battling each other, but Mylonakis warns against a simplistic extrapolation of these results to higher organisms. "The worm dies slower [in the co-infection model]," he says, "but that is not what we see in patients. In humans we see a delay in the development of respiratory infection and then [...] a more severe infection. Having two pathogens in your airways is not necessarily better than having only one."

Through the course of evolution the two pathogens have learned to deal with each other, and these are lessons we need to learn to help combat problematic infections. Nicole Rusk

\section{RESEARCH PAPERS}

Peleg, A.Y. et al. Prokaryote-eukaryote interactions identified by using Caenorhabditis elegans. Proc. Natl. Acad. Sci. USA 105, 14585-14590 (2008). 